Apidologie, 1988, 19 (3), 307-318

\title{
A STUDY OF RAPESEED (BRASSICA NAPUS L. VAR. OLEIFERA METZGER) FLOWER NECTAR SECRETIONS
}

\author{
Jacques MESQUIDA *, René MARILLEAU **, Minh-Hà PHAM-DELEGUE **, \\ Michel RENARD *** \\ * I.N.R.A., Centre de Recherches de Rennes \\ Laboratoire de Zoologie \\ Domaine de la Motte au Vicomte \\ B.P. 29, F 35650 LE RHEU \\ ** I.N.R.A., C.N.R.S. \\ Laboratoire de Neurobiologie Comparée des Invertébrés \\ La Guyonnerie \\ F 91440 BURES-SUR-YVETTE \\ *** I.N.R.A., Centre de Recherches de Rennes \\ Station d'Amélioration des Plantes \\ Domaine de la Motte au Vicomte \\ B.P. 29, F 35650 LE RHEU
}

\begin{abstract}
SUMMARY
Two nectar extraction techniques, micropipetting and centrifugation, were compared to establish a reliable basis for analysis and comparison of nectar production of rapeseed lines under selection. All tests used Kid cultivar. Apart from quantitative measurements for nectar production, nectar glucid composition was assessed by gas chromatography. Centrifugation provided larger quantities of liquid $(\times 4-6)$, but total glucid content was not greater than for micropipetting. Centrifugation thus artificially diluted nectar and produced samples unrepresentative of those actually encountered by insects. Glucose and fructose fractions were very similar using either method. Micropipetting is therefore recommended for future work. Such sampling may provide the basis for selecting oilseed rape lines for nectar quantity and quality to optimize bee visits and cross pollination.
\end{abstract}

\section{INTRODUCTION}

Oilseed rape (Brassica napus L. var oleifera METZGer) is partially autogamous (70\%, SYlveN, 1920 ; RIVES, 1957 ; MORICE, 1960 ; RUDLOFF \& SCHWEIGER, 1984), but its abundant nectar and pollen attract numerous insect pollinators. Various studies of rapeseed pollinating agents have indicated that the plant benefits from insect visitation (FrEe \& NUTTALL, 1968; Williams, 1978) despite the high efficiency of wind pollination (Olsson, 1955 ; 
Mesquida \& Renard, 1981, 1982). Insect pollination has more effect on plant phenology than on seed production (RENARD \& MESQuida, unpublished data). Better understanding of the role of pollinating insects and factors affecting plant insect relations is of great importance to $\mathrm{F} 1$ hybrid seed production using male sterile lines.

Field experiments have shown that honeybees (Apis mellifera L.) are the main pollinators (TASEI, 1977 ; Mesquida \& ReNARD, 1978, 1979 (a), 1979 (b) ; RENARD \& MESQuidA, 1983). Moreover, foraging honeybees may show a preference for male fertile lines as opposed to male sterile lines (cytoplasmic « OGURA » type male sterility). The difference may reflect lower nectar secretion in these male sterile lines. Quality and quantity of nectar production have been shown to be significant for selective foraging behavior in honeybees (Masson, 1983).

Therefore, reliable techniques for assessing nectar production of selected lines are needed if entomophilous pollination is to be fully assessed and exploited in selecting F1 hybrid seed parental lines. The objective is to equalize bee distribution between male sterile and male fertile lines in field crops.

This paper compares nectar collection from rape flowers using centrifugation versus glass micropipettes. In addition to quantitative analysis of nectar production, nectar glucidic component was also determined. The method used for glucidic composition analysis was derived from one previously applied to sunflower and oilseed rape (Fonta et al., 1985 ; Le Metayer pers. comm.).

\section{MATERIALS AND METHODS}

\section{Plant material}

All experiments were done using a winter cultivar Kid, at the I.N.R.A. experimental station (Rennes, France). Samples were collected in the morning ( $8 \mathrm{~h}$ GMT) on freshly opened flowers, on two dates in April 1983. Plants in full flower were bagged $24 \mathrm{hrs}$ prior sampling to prevent nectar collection by foraging insects.

\section{Nectar extraction by centrifugation}

Nectar was extracted from five flowers per plant on 15 April (first collection, 20 plants) and on 20 April (second collection, 10 plants). Sampling and centrifugation at $3000 \mathrm{r.m}$. followed methods described by Bosi (1973).

Nectar extract weight was calculated from the difference in weight before and after centrifugation.

Nectar extraction using micropipettes

Glass micropipettes (internal diameter $1 \mathrm{~mm}$, volume $5 \mu 1$.), were used to obtain samples from up to 5 flowers per plant from 20 plants in the first collection and from 10 plants in the second collection. 
Volumes of nectar collected were read directly from micropipette scales, and samples were then weighed before freeze storage $\left(-25^{\circ} \mathrm{C}\right)$ for later analyses.

After micropipette collection, up to 5 flowers from 6 plants were subjected to centrifugation in the second collection.

\section{Analysis of nectar glucid composition}

\section{a) Sample preparation}

Nectar was partially dehydrated under vaccum overnight in the presence of di-phosphorous pentoxide, after determining flower nectar weight. Trimethylsilylesters were prepared following BROHST \& LOTT (1966) and BosI (1973), the sample being dehydrated in pyridine (approximately $1 \mathrm{ml}$ ) with addition of $0.9 \mathrm{ml}$ of hexamethyldisilazane and $0.1 \mathrm{ml}$ of trifluoroacetic acid in that order. The solution was shaken for a few minutes, then left for 12 hours to ensure complete derivation of trisaccharides.

\section{b) Preparation of standard reference solutions}

Two $\mathrm{mg}$ of reference sugars (glucose, fructose or sucrose) were dissolved in $1 \mathrm{ml}$ of pyridine, and dehydrated as for samples, so that sugars were then at $1 \mu \mathrm{g} / 1$ concentrations.

\section{c) Chemical analysis}

A GIRDEL 75 gas chromatograph equipped with a pyrex glass column $(3 \mathrm{~m} \times 2 \mathrm{~mm})$ was used, filled with a stationary phase, type OV 17 at $3 \%$ on Chromosorb WHP. Column temperature was programmed from $185^{\circ} \mathrm{C}$ to $280^{\circ} \mathrm{C}$, at $2^{\circ} \mathrm{C} / \mathrm{min}$. Detector temperature was $300^{\circ} \mathrm{C}$. Carrier gas was nitrogen at 2 $\mathrm{kg} / \mathrm{cm}^{2}$ and a $24 \mathrm{ml} / \mathrm{min}$ flow.

Detector signals were treated by a Housron integrator recorder, recording speed $5 \mathrm{~mm} / \mathrm{min}$. Injected volumes were $2 \mu \mathrm{l}$. Standard solution was injected after each series of 4 or 5 samples.

d) Calculation of sugar proportions (following BosI, 1973)

\section{i. Proportion of dry weight attributable to each sugar}

Pi $\%=\frac{\text { Ai fi } \times 100}{\Sigma A i \times f i}$

Ai : peak area of a given sugar in standard solution ;

fi : response coefficient calculated from a standard solution, relative to one of the reference sugars.

$\mathrm{fi}=\frac{\mathrm{Ar}}{\mathrm{A}^{\prime} \mathrm{i}}$

Ar: area of reference sugar peak in standard solution ;

$A^{\prime} i$ : area of sample sugar in standard solution.

\section{ii. Total sugar concentration}

Considered to be equal to nectar dry weight since amino acid and other nectar components represent less than $0.03 \%$ of nectar dry weight for most flower nectars (BAKER, cited by Heinrich, 1975).

Nectar dry weight was obtained from

$\mathrm{C}$ total $\%=\Sigma \frac{\mathrm{Ai}}{\mathrm{A}^{\prime} \mathrm{i}} \times \frac{\mathrm{V}}{\mathrm{P}} \times 100$

$\mathrm{V}=$ dilution volume ;

$\mathbf{P}=$ nectar weight prior to dehydration. 
iii. Concentration of each sugar in nectar:

$\mathrm{Ci} \%=\frac{\mathrm{Ai}}{\mathrm{A}^{\prime} \mathrm{i}} \times \frac{\mathrm{V}}{\mathrm{P}} \times 100$

Data treatment

Effects of sampling method and sampling date, respectively, were analyzed using Student's t test after checking for applicability (DAGNELIE, 1970).

\section{RESULTS}

\section{Nectar production}

Nectar weight per flower (mg) is shown in Table 1. Centrifugate sample volumes were always 4 to 6 times greater than for micropipettes. Sample volumes obtained using micropipettes were similar for the two collection dates, but differed significantly for centrifugated samples.

TABL. 1. - Mean weight ( \pm standard error) (mg) of nectar/flower according to method and collection date, 1983 .

\begin{tabular}{|c|c|c|c|}
\hline Date & Micropipette collection & Centrifugation & Method effect \\
\hline 15 April & $\begin{array}{l}0.80( \pm 0.16) \\
9\end{array}$ & $\begin{array}{l}4.77( \pm 0.21) \\
10\end{array}$ & $* * *$ \\
\hline 20 April & $\begin{array}{l}0.68( \pm 0.16) \\
6\end{array}$ & $\begin{array}{l}2.91( \pm 0.11) \\
9\end{array}$ & $* * *$ \\
\hline Date effect & n.s. & $* * *$ & \\
\hline
\end{tabular}

Significance level as determined by Student's t-test

n.s. : not significant

* : $\mathrm{p}<0.05$

**: $\mathrm{p}<0.01$

$* * *: \mathrm{p}<0.001$

\section{Glucid content}

The two principle sugars identified by chromatographic analyses were glucose and fructose.

\section{- Nectar sugar concentration}

Sugar concentration in nectar sampled by micropipette was between $40 \%$ and $50 \%$ (Table $2 \mathrm{a}$ ). Concentration was about one fourth as high in centrifu- 
gated samples, and this was also so for each sugar considered separately (tables $2 b \& 2 c$ ). There was no significant effect of sample date for either extraction method.

TABL. 2. - Mean concentrations ( \pm standard error) (\%) of nectar according to method and collection date, 1983.

\begin{tabular}{|c|c|c|c|c|}
\hline & Date & $\begin{array}{l}\text { Micropipette } \\
\text { collection }\end{array}$ & Centrifugation & Method effect \\
\hline \multirow{3}{*}{$\begin{array}{l}\text { a } \\
\text { Total sugars } \\
\text { or dry matter }\end{array}$} & 15 April & $41.08( \pm 2.70)$ & $11.21( \pm 1.44)$ & $* * *$ \\
\hline & 20 April & $48.05( \pm 5.27)$ & $13.45( \pm 0.88)$ & $* * *$ \\
\hline & Date effect & n.s. & n.s. & \\
\hline \multirow{3}{*}{$\begin{array}{c}\mathrm{b} \\
\text { Fructose }\end{array}$} & 15 April & $19.61( \pm 1.41)$ & $5.57( \pm 0.74)$ & $* * *$ \\
\hline & 20 April & $23.38( \pm 2.58)$ & $6.72( \pm 0.42)$ & $* * *$ \\
\hline & Date effect & n.s. & n.s. & \\
\hline \multirow{3}{*}{$\begin{array}{c}c \\
\text { Glucose }\end{array}$} & 15 April & $27.47( \pm 1.34)$ & $5.65( \pm 0.70)$ & $* * *$ \\
\hline & 20 April & $25.35( \pm 2.96)$ & $6.73( \pm 0.46)$ & $* * *$ \\
\hline & Date effect & n.s. & n.s. & \\
\hline
\end{tabular}

Significance level as determined by Student's t-test n.s. : not significant

* $\mathrm{p}<0.05$

$* *: p<0.01$

$* * *: \mathrm{p}<0.001$

TABL. 3. - Mean relative dry weight concentrations ( \pm standard error) (\%) of sugars, according to method and collection date, 1983.

\begin{tabular}{c|c|c|c|c}
\hline \multirow{2}{*}{$\begin{array}{c}\text { a } \\
\text { Fructose }\end{array}$} & Date & $\begin{array}{c}\text { Micropipette } \\
\text { collection }\end{array}$ & Centrifugation & Method effect \\
\cline { 2 - 5 } & 15 April & $47.29( \pm 0.85)$ & $48.48( \pm 0.53)$ & n.s. \\
\cline { 2 - 5 } & 20 April & $48.65( \pm 0.40)$ & $49.90( \pm 0.36)$ & $*$ \\
\hline \multirow{3}{*}{ blucose } & 15 April & $52.71( \pm 0.85)$ & $51.53( \pm 0.52)$ & n.s. \\
\cline { 2 - 5 } & 20 April & $51.35( \pm 0.40)$ & $50.10( \pm 0.35)$ & $*$ \\
\cline { 2 - 5 } & Date effect & n.s. & $*$ & $*$ \\
\hline
\end{tabular}

Significance level as determined by Student's t-test

n.s. : not significant

$\because \mathrm{p}<0.05$

$\because \mathrm{p}<0.01$

$* * *: p<0.001$ 


\section{- Sugar proportions-dry weight}

Glucoce (Table 3) content was higher than that of fructose by about $3 \%-4 \%$. There were slight differences in estimates for different sugars with date and method of collection, but no significant, except for centrifugated samples in the second collection.

\section{- Sugar content in nectar produced per flower}

Table 4 shows no significant difference between either dates or sampling methods for dry weights, or fructose or glucose contents. Total sugar weight in nectar produced per flower was approximately $0.4 \mathrm{mg}$ (Table 4a).

TABL. 4. - Mean weights ( \pm standard error) (mg) of sugars in the nectar/flower, according to method and collection data, 1983.

\begin{tabular}{|c|c|c|c|c|}
\hline & Date & $\begin{array}{l}\text { Micropipette } \\
\text { collection }\end{array}$ & Centrifugation & Method effect \\
\hline \multirow{3}{*}{$\begin{array}{c}\text { a } \\
\text { Total sugars } \\
\text { of dry matter }\end{array}$} & 15 April & $0.37( \pm 0.06)$ & $0.51( \pm 0.07)$ & n.s. \\
\hline & 20 April & $0.30( \pm 0.04)$ & $0.40( \pm 0.03)$ & n.s. \\
\hline & Date effect & n.s. & n.s. & \\
\hline \multirow{3}{*}{$\begin{array}{c}\mathrm{b} \\
\text { Fructose }\end{array}$} & 15 April & $0.18( \pm 0.03)$ & $0.26( \pm 0.03)$ & n.s. \\
\hline & 20 April & $0.14( \pm 0.02)$ & $0.18( \pm 0.02)$ & n.s. \\
\hline & Date effect & n.s. & n.s. & \\
\hline \multirow{3}{*}{$\begin{array}{c}\text { c } \\
\text { Glucose }\end{array}$} & 15 April & $0.19( \pm 0.03)$ & $0.27( \pm 0.03)$ & n.s. \\
\hline & 20 April & $0.15( \pm 0.02)$ & $0.20( \pm 0.02)$ & n.s. \\
\hline & Date effect & n.s. & n.s. & \\
\hline
\end{tabular}

Significance level as determined by Student's t-test n.s. : not significant

* : $\mathrm{p}<0.05$

**: $\mathrm{p}<0.01$

$* * *: p<0.001$

\section{- Centrifugation after micropipette collection}

Although an appreciable amount of liquid was extracted by centrifugation after micropipette sampling (Table 5a), the weight and concentration of sugars extracted were negligible (Tables 5b-g). 
TABL. 5. - Quantity and composition of nectars obtained from centrifugation following micropipette collection

\begin{tabular}{|c|c|c|}
\hline & Micropipette collection & \begin{tabular}{|c} 
Centrifugation following \\
micropipette collection
\end{tabular} \\
\hline Nectar weight/flower $(\mathrm{mg})$ & $0.68( \pm 0.16)$ & $1.42( \pm 0.36)$ \\
\hline $\begin{array}{l}\mathrm{b} \\
\text { Fructose weight in nectar/flower }(\mathrm{mg})\end{array}$ & $0.14( \pm 0.02)$ & $0.01( \pm 0.01)$ \\
\hline $\begin{array}{c}\mathrm{c} \\
\text { Glucose weight in nectar/flower (mg) }\end{array}$ & $0.15( \pm 0.02)$ & $0.01( \pm 0.01)$ \\
\hline $\begin{array}{c}\text { d } \\
\text { Total nectar dry weight }(\mathrm{mg})\end{array}$ & $0.29( \pm 0.04)$ & $0.02( \pm 0.01)$ \\
\hline$\%$ fructose in nectar & $23.38( \pm 2.58)$ & $0.67( \pm 0.32)$ \\
\hline$\%$ glucose in nectar & $25.35( \pm 2.96)$ & $0.53( \pm 0.24)$ \\
\hline $\begin{array}{c}\mathrm{g} \\
\% \text { dry matter in nectar } / \text { flower }\end{array}$ & $48.05( \pm 5.27)$ & $1.20( \pm 0.56)$ \\
\hline
\end{tabular}

Significance level as determined by Student's t-test n.s. : not significant

$*: \mathrm{p}<0.05$

$* *: \mathrm{p}<0.01$

*** $: \mathrm{p}<0.001$

\section{DISCUSSION}

The whole sugar fraction of nectar was obtained by micropipetting, although four to six times more liquid was extracted by centrifugation. Consequently, centrifugation diluted sugar fractions in samples relative to real content. An effect on collection method on estimation of nectar production was already noted for sunflower by MAdeuf (pers. comm.) and GirNik (1976) who preferred micropipette collection to both centrifugation and washing. Other methods for measuring nectar secretion have been described. Swanson and SHuEl (1949) considered a volumetric centrifugation method to be reliable for yield data with large scale sampling. LivTzeva (1954) compared several nectar collection methods, and concluded that filter paper was least reliable (due to evaporation) as compared with pipette or washing methods. Water extraction methods seem to be seriously limited (von PlantA, 1886 ; BonNIER, 
1893 ; KENOYER, 1917), since extract sugar content may include sugars lixiviated from plant tissue cells. Most authors consider micropipette collection to be preferable, since it requires only the simplest equipment and is reliable (VAnsell et al., 1942 ; Vansell, 1943, 1944 ; Swanson and Shuel, 1949).

Proportions of fructose and glucose in Kid variety nectar were very similar, but no trace of sucrose was found, althought the latter has been identified at low titres in other oilseed rape varieties (PERCIVAL, 1961).

Such differences in estimates of nectar glucid composition may be related to differences in sensitivity of gas chromatography techniques applied, but a varietal effect cannot be excluded. It might then be possible to detect and define nectar glucidic compositions specific to oilseed rape genotypes as suggested by FONTA et al. (1985) in reference to sunflower. Since nectar sugar content, and notably that of sucrose can be critical for bee foraging preferences (Pham-Delegue et al., 1985), screening of nectars of male fertile and male sterile lines for both sugar content and glucid composition may contribute to optimization of cross pollination.

In conclusion, even though no single method is satisfactory for all plants, nectar collection using micropipettes is preferred to centrifugation for study of rapeseed flower nectars. The latter provides larger sample quantities, but these are unrepresentative of nectar actually accessible to insects, and modification of chemical composition, due to lesion of plant tissues cannot be excluded. Micropipette nectar collection offers the best available sampling method, for assessing quantitative and qualitative aspects of nectar production as functions of genotype and phenological state. It may also be used to determine pedoclimatic influences on nectar secretion.

Received for publication in October 1987. Accepted for publication in April 1988.

\title{
ACKNOWLEDGMENTS
}

The authors wish to thank Dr. C. MASson for a critical review of the manuscript and M. LE METAYER for helpful support in data treatment.

\author{
RÉSUMÉ \\ ÉTUDE DES SÉCRÉTIONS NECTARIFĖRES DES FLEURS DE COLZA \\ (BRASSICA NAPUS L. VAR. OLEIFERA METZGER)
}

La pollinisation du colza (Brassica napus L. var. oleifera METzGER), assurée en partic par le vent, dépend toutefois de laction des insectes pollinisateurs, notamment dans le cadre de la production de semences hybrides F1. Chez cette plante, la faune pollinisatrice est principalement constituée d'abeilles 
domestiques; celles-ci manifestent des préférences génotypiques chez certaines lignées mâles-stériles qui pourraient être liées à des différences de sécrétions nectarifères. Afin de disposer d'une technique fiable d'estimation et de comparaison des productions nectarifères de différentes lignées en cours de sélection, deux techniques d'extraction des nectars, par centrifugation et par pipetage, ont été comparées pour les nectars de la variété Kid. Outre une mesure quantitative des productions nectarifères, une méthode d'analyse par chromatographie en phase gazeuse des constituants glucidiques a été appliquée. La méthode d'extraction par centrifugation permet de recueillir des quantités de solution plus abondantes (4 à 6 fois) que par pipetage (Tabl. 1) ; toutefois la totalité de la fraction glucidique représentative des nectars est intégralement présente dans des solutions prélevées par pipetage (Tabl. 5) : les sucres sont donc dilués par centrifugation. La présence de glucose et de fructose, en proportions voisines, a été mise en évidence (Tabl. 2, 3, 4). L'adoption de la technique de prélèvement par pipetage, qui assure une restitution plus fidèle des sécrétions disponibles pour les insectes pollinisateurs, est préconisée et pourrait permettre la sélection de lignées en vue d'une optimisation de la pollinisation croisée.

\section{ZUSAMMENFASSUNG}

\section{UNTERSUCHUNGEN ÜBER DIE BLÜTENNEKTARSEKRETION VON RAPS (BRASSICA NAPUS L. VAR. OLEIFERA METZGER)}

Raps (Brassica napus L. var oleifera MEtzGer), wird zum größten Teil vom Wind bestäubt. Jedoch hängt vor allem die Produktion von F1 Hybrid-Samen von der Aktivität bestäubender Insekten ab. Hierbei spielt die Honigbiene die größte Rolle. Die Nektar sammelnden Honigbienen zeigen eine Präferenz für männlich-fertile Linien, was dadurch zustande kommen könnte, daß Unterschiede in der Nektarsekretion bestehen. Um die im Laufe der Selektion entstandenen Unterschiede in der Nektarsekretion mit einer verläßlichen Methode zu schätzen, wurden zwei Extraktionsmethoden, die Zentrifugation und dic Pipettierung (anhand des Nektars der Varietät Kid) verglichen. Über die quantitative Messung der Nektarproduktion hinaus wurde eine gaschromatographische Analyse der Kohlehydrat-Komponenten durchgeführt.

Die Methode der Zentrifugation führt zu einer größeren Ausbeute (4 bis 6 mal) als die Pipettierung (Tab. 1). Dennoch war die Gesamtheit der repräsentativen Kohlenhydrat-Fraktion auch in den pipettierten Proben vorhanden, was darauf schließen läßt, daß in den zentrifugierten Proben die Zucker nur mehr verdünnt sind. Es konnte gezeigt werden, daB Glukose und Fruktose in ähnlichen Proportionen vorliegen (Tab. 2, 3 u. 4).

Die Methode des Pipettierens eignet sich besser, wenn man analysieren will, welche Sekretionsmenge dem bestäubenden Insekt effektiv zur Verfügung steht. Außerdem könnte sie die Selektion von Linien im Hinblick auf eine Optimierung der Kreuzbestäubung begünstigen.

\section{REFERENCES}

Bonnier G., 1893. - Influence du terrain sur la production du nectar des plantes. Assoc. Fr. Av. Sci., 2, 567-569.

Bosı G., 1973. - Méthode rapide pour la détermination par chromatographie en phase gazeuse des glucides du nectar : technique de prélèvement du nectar et de préparation des éthers triméthylsilylés en présence d'cau. Apidologie, 4 (1), 57-64. 
Brohst K.M., LotT C.E., 1966. - Determination of some components in corn symp by gas liquid chromatography of the trimethylsilyl derivatives. Cereal Chem., 43, 35-43.

Dagnelie P., 1970. - Théorie et méthodes statistiques. Applications agronomiques. 2 vol., Duculot, S.A., Gembloux, 378 p. et 451 p.

Fonta C., Pham-Delegue M.H., Marilleau R., Masson C., 1985. - Rôle des nectars de tournesol dans le comportement des insectes pollinisateurs et analyse qualitative et quantitative des éléments glucidiques de ces sécrétions. Acta Ecologica, EEcol. Applic., 6 (2), 175-186.

Free J.B., Nuttall P.M., 1968. - The pollination of oilseed rape (Brassica napus) and the behaviour of bees on the crop. J. Agric. Sci. Camb., 71, 91-94.

Girnik D., 1976. - [Productivité en nectar du tournesol]. [En russe]. Pchelovodstvo, 8, 10-11.

Livtzeva E.K., 1954. - [Methods used in nectar secretion investigations]. [In Russian]. Pchelovodstvo, 31 (11), 33-39.

HeinRich B., 1975. - Energetics of pollination. Annu. Rev. Ecol. Syst., 6, 139-170

KeNOYER L.A., 1917. - Environmental influences on nectar secretion. Bot. Gaz, 63, 249-365.

Masson C., 1983. - Rôle des médiateurs chimiques d'origine animale et végétale dans la pollinisation. C.R. $5^{e}$ Symp. Int. Pollinisation, Versailles, sept. 1983, 25-37.

Mesquida J., Renard M., 1978. - The entomophilous pollination of male sterile strains of winter Rapeseed (Brassica napus L. var. oleifera METZGER) and a preliminary study of alternating devices. Proc. 4th. Int. Symp. Pollination, Maryland, 49-57.

Mesquida J., Renaro M. 1979 (a). - Importance de l'abeille domestique (Apis mellifica L.) sur la pollinisation du colza d'hiver mâle-stérile (Brassica napus L. var. oleifera Metzger) en production de semences hybrides F1 et conséquences sur les dispositifs d'alternance. Symp. Pollinisation ITAPIAPIMONDIA, Avignon, 49-60.

Mesquida J., Renard M., 1979 (b). - Résultats préliminaires sur la pollinisation du colza d'hiver mâlestérile et les modalités de production de semences hybrides. Inform. Tech. CETIOM, , 65, 4-14.

Mesquida J., Renard M., 1981. - Pollinisation du colza d'hiver mâle-fertile et mâle-stérile (Brassica napus L. var. oleifera MEtzGer) par l'abeille domestique (Apis m. mellifica L.) : effets sur la phénologie et le rendement. Apidologie, 12, 345-362.

Mesquida J., Renard M., 1982. - Etude de la dispersion du pollen par le vent et de l'importance de la pollinisation anémophile chez le colza (Brassica napus L. var. oleifera Metzger). Apidologie, 13 (4), 353-366.

Morice J., 1960. - La sélection du colza d'hiver basée sur l'étude des composantes du rendement. Ann. Amélior. Plantes, 1, 85-116.

Otsson G., 1955. - Vindpdlinering hos korsblomstriga oljeväxter. (in Swedish, English Summary), Sver. Utsadesfoi., 65, 418-422.

Percival M.S., 1961. - Types of nectar in Angiosperms. New Phytol, 60, 235-281.

Pham-Delegue M.H., Masson C., Bonjean A., Etievant P., Marilleau R., 1985. - Etude des paramètres chimiques impliqués dans la pollinisation du tournesol par les abeilles en production de semences hybrides. C.R. XI Conf. Int. Tournesol, Mar del Plata (Argentine), mars 1985, 559-564.

Planta A. von, 1886. - Über die Zusammensetzung einiger Nektar-Arten. Z. Physiol. Chem., 10, 227247.

Renard M., Mesquida J., 1983. - Pollinisation entomophile du colza mâle-stérile en production de semences hybrides F1 dans différentes régions de France. C.R. VI congr. Int. sur le Colza, Paris, $552-557$.

Rives M., 1957. Etudes sur la sélection du colza d'hiver. Ann. Amélior. Plantes, 1, 61-107.

Rudloff E., Schweiger W., 1984. - Eine Methode zur Schätzung der Fremdbefruchtungsrate bei Qualitätsraps (Brassica napus L.). Arch. Züchtungsforsch., Berlin, 14, 327-334.

Swanson C.A., Shuel R.W., 1950. - The centrifuge method for measuring nectar yield, Plant Physiol., 25 (3), 513-520.

SyLven N., 1920. - Om självoch Korsbefrukting hos rapsen. Sver. Utsädesför. T., 6, 225-244. 
TASEI J.N., 1977. - Observations sur le colza d'hiver-floraison-sécrétion nectarifère-visite par les abeilles. Bull. tech. Apic., 4, 9-16.

VANSEll G.H., Watkins W.Q., Bishop R.K., 1942. - Orange nectar and pollen in relation to bee activity. J. econ. Entomol., 35, 321-323.

VANSEll G.H., 1943. - Alfalfa nectar and the honey bee. Am. Bee J., 83, 106-107.

VANSEll G.H., 1944. - Cotton nectar in relation to bee activity and honey production. J. econ. Entomol., 37, 528-530.

Williams I.H., 1978. - The pollination requirements of swede rape (Brassica napus L.) and of turnip rape (Brassica campestris L.). J. Agric. Sci., Camb., 91, 343-348. 